\title{
A Preliminary Study on the Usability of Head Mount Display (HMD) for Evaluating Attentional Behavioral Disturbance
}

\author{
Tsutomu Sasaki, OTR, PhD ${ }^{1,2}$, Hisae Kitajima, OTR, PhD², \\ Kunihiro Kanaya, OTR, MS4,5, Kosuke Sugama, OTR, MS ${ }^{6}$, \\ Yasuhito Sengoku, OTR, $\mathrm{PhD}^{7}$ \\ ${ }^{1}$ Division of Occupational Therapy, Shinshu University \\ 2 Visiting Research Fellow of Sapporo Medical University \\ ${ }^{3}$ Department of Occupational Therapy, Hokkaido Bunkyo University \\ ${ }^{4}$ Graduate School of Health Sciences, Sapporo Medical University \\ ${ }^{5}$ Department of Rehabilitation Medicine, Sasson-Sugata Clinic \\ ${ }^{6}$ Department of Rehabilitation, Nishi Otaru Hospital, Midori-no-Sato Institution for the Severe Motor and Intellectual \\ Disabilities(SMID) \\ 7 Department of Occupational Therapy, Sapporo Medical University
}

\begin{abstract}
To investigate the clinical applicability of head-mount display (HMD) in evaluating attentional behavioral disorders, we developed a new desk-task environment with HMD. 18 patients with right hemisphere damage were recruited. We compared the results of traditional neuropsychological tests and tasks carried out under the HMD setting using attention behavioral disturbances observed in daily life. The findings show that performance under HMD settings predicts the presence or absence of attention behavioral disturbance with higher accuracy than traditional tasks. Our newly developed HMD system shows promise as an appropriate method for assessing attentional behavioral disturbances. Although necessarily speculative at this stage, we believe that the more dynamic cognitive demands required to successfully accomplish the HMD task might be linked to attentional behavior in ADL.
\end{abstract}

Key words: stroke, attention, head-mount display (HMD)

(Asian J Occup Ther 8: 21-29, 2010)

\section{Introduction}

The purpose of Occupational Therapy (OT) is to help people increase their functional independence in daily life while preventing or minimizing disability. Physical, psychological, and cognitive factors, as well as personal history, are linked causally to disabilities. In the clinical setting, some patients show behavioral disorders mainly due to cognitive deficits, and occupational therapists often apply several neuropsychological tests to verify the factors causing their behavioral disorders. However, it has been recognized that some patients don't show any abnormalities in neuropsychological tests, but do display behavioral

Received: 21 December 2009, Accepted: 15 August 2010

Corresponding to: Tsutomu Sasaki, Division of Occupational Therapy, Shinshu University, 3-1-1 Asahi, Matsumoto, Nagano 390-8621, Japan e-mail: tusasaki@shinshu-u.ac.jp

(C2010 Japanese Association of Occupational Therapists deficits in activities of daily living (ADL) (Sasaki, Sengoku, Nakajima, Sugama \& Kitajima, 2005). The authors investigated the relation between results of a revised bisection task and attentional performance in ADL in stroke patients. The results showed that some patients exhibited no behavioral abnormalities in spite of their lower score in the task.

This clinical discrepancy has a negative impact on our clinical practice because it restricts our understanding of the impairments causing patients' behavioral disorders. This in turn can lead to anomalies in communication with other medical staff and even patients' families, resulting in disagreements regarding treatment options. Accordingly, evaluations with higher accuracy in detecting behavioral abnormalities should be developed.

We assume that one of the reasons for these discrepancies could be environmental differences: static in desktask settings vs. dynamic in ADL settings. In general, it 
Table 1. Demographic and clinical characteristics

\begin{tabular}{|c|c|c|c|c|c|c|c|c|c|c|}
\hline case & age & $\operatorname{sex}$ & etiology & $\begin{array}{l}\text { duration from } \\
\text { onset (month) }\end{array}$ & FIM & MMSE & motor deficit & $\begin{array}{c}\text { sensory } \\
\text { deficit }\end{array}$ & USN & $\begin{array}{c}\text { visual field } \\
\text { deficit }\end{array}$ \\
\hline 1 & 62 & female & infraction & 11 & 95 & 23 & moderate & +++ & + & + \\
\hline 2 & 72 & male & infraction & 1 & 87 & 24 & mild & + & + & + \\
\hline 3 & 76 & male & infraction & 1 & 70 & 28 & mild & + & + & + \\
\hline 4 & 77 & male & infraction & 2 & 103 & 28 & mild & - & + & + \\
\hline 5 & 58 & male & infraction & 1 & 101 & 20 & mild & + & + & + \\
\hline 6 & 54 & male & hemorrhage & 3 & 71 & 18 & severe & +++ & + & + \\
\hline 7 & 55 & male & hemorrhage & 5 & 77 & 26 & severe & ++ & + & + \\
\hline 8 & 57 & female & infraction & 1.5 & 74 & 27 & severe & ++ & + & - \\
\hline 9 & 62 & male & hemorrhage & 1 & 91 & 28 & severe & +++ & + & - \\
\hline 10 & 57 & male & infraction & 4 & 101 & 29 & severe & +++ & + & - \\
\hline 11 & 61 & female & hemorrhage & 1 & 119 & 26 & mild & ++ & - & - \\
\hline 12 & 69 & male & hemorrhage & 3 & 114 & 29 & moderate & + & - & - \\
\hline 13 & 75 & female & infraction & 1 & 110 & 25 & mild & + & - & - \\
\hline 14 & 78 & male & infraction & 0.5 & 126 & 30 & mild & - & - & + \\
\hline 15 & 58 & male & infraction & 1 & 63 & 25 & severe & +++ & - & - \\
\hline 16 & 57 & male & infraction & 2.5 & 115 & 29 & moderate & +++ & - & - \\
\hline 17 & 74 & male & infraction & 0.5 & 99 & 30 & mild & + & - & - \\
\hline 18 & 50 & male & infraction & 1 & 122 & 30 & mild & + & - & - \\
\hline ave. & 64.0 & & & 2.3 & 96.6 & 26.4 & & & & \\
\hline $\mathrm{SD}$ & 8.9 & & & 2.4 & 18.9 & 3.3 & & & & \\
\hline
\end{tabular}

a. sensory deficit; +++, severe; ++, moderate; +, mild; -, absent. b. USN; +, present; -, absent. c. visual field deficit; +, present; -, absent.

has been thought that the desk-task setting is so static that less sensory integration was needed to successfully accomplish a given task (Thompson \& Sanderson, 2008). Another reason, postulated by Deouell, Sacher, and Soroker (2005), was that desk tasks do not reflect the dynamic character of the natural daily environment, in which relevant stimuli (e.g. background of objects and changing shadow) occur. That is, in most cases, patients sit and carry out relatively one-dimensional tasks, e.g. vision for a search task. Patients no longer need to process stimuli irrelevant to desk tasks and only less sensory information has to be integrated to successively accomplish tasks. On the other hand, ADL settings involve dynamic situations that require patients to integrate external and internal incoming sensory information properly while performing concurrent activities. Few studies have been reported in which the subjects were required to concentrate on environmental differences while performing desk-tasks, and in which the relationship between the results of desk tasks and ADL performance was analyzed.

Hence, we developed a desk-task setting using a head mount display (HMD) and a tablet, in which more sensory integration is needed than in the traditional desk-task setting. The present work is intended to evaluate the clinical applicability of the test settings.

\section{Materials and Methods}

\section{Subjects}

18 patients (14 men and 4 women) with right hemisphere damage were the subjects of this study. Participants gave written informed consent, and the experiments were approved by the local ethics committee of Sapporo Medical University. All subjects had undergone a usual rehabilitation program. The mean age was 64.0 with a standard deviation of 8.9. The score of the Mini-Mental States of Examination (MMSE) ranged from 18 to 30 and its average $\pm S D$ was $26.4 \pm 3.3$. The mean months from onset were $2.3 \pm 2.4$ (ranging from 0.5 to 11 ). 8 out of 18 subjects had visual field deficit on confrontation. 10 patients exhibited spatial neglect in at least one task including cancellation, copying, bisection, and behavior in daily life. We excluded subjects who had severe dementia, aphagia, or apraxia as well as those who couldn't concentrate on successive tasks for about 45 minutes. The clinical and demographic data are summarized in Table 1. Patients who were mild to moderate in their severity were selected because the discrepancy between results of desk tasks and performance in ADL settings (proposed in the introduction) occurs in mild patients.

\section{Tasks in traditional settings}

Subjects were seated in front of a table, on which a task sheet was placed in front of their trunk midline. 


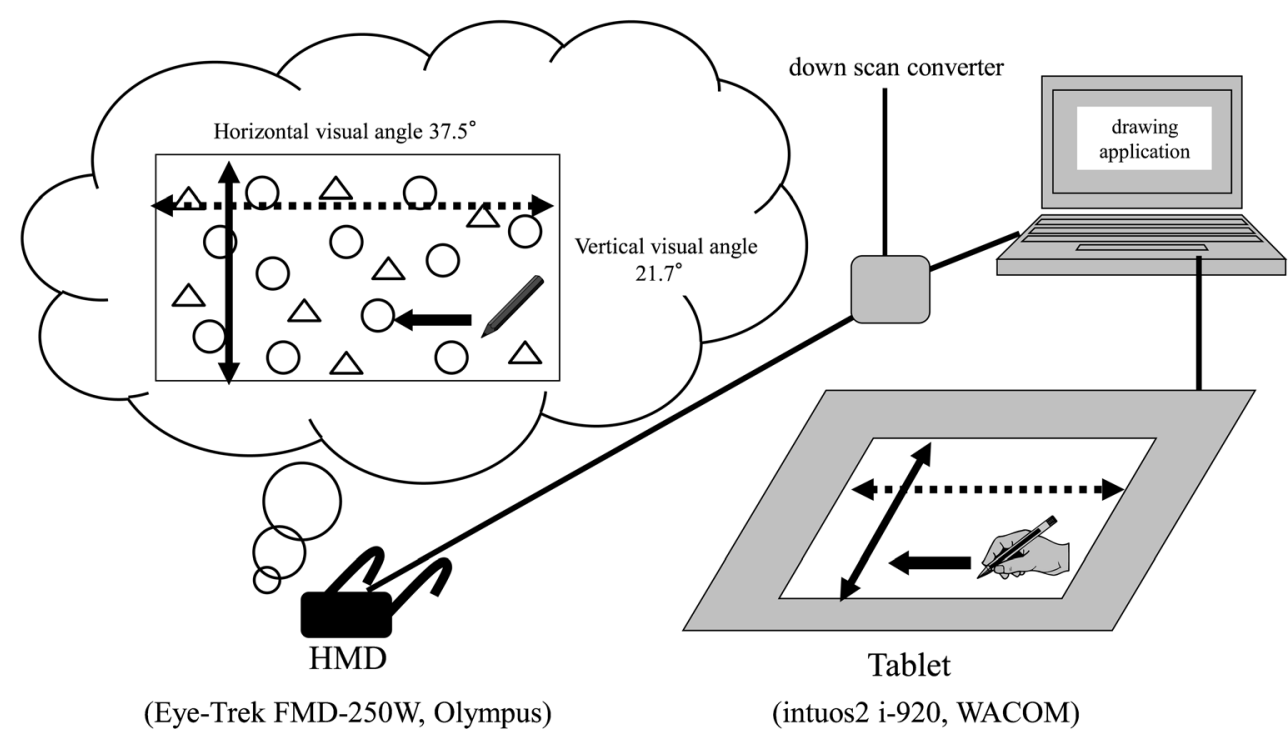

Fig. 1. Schematic representation of experimental setting in HMD environment. In HMD setting, the tablet is placed in front of subjects. Subjects wear HMD during the tasks. Subjects see only a task stimulus, pen icon and the marks they make on the HMD display by manipulating the pen on the tablet. There is no apparent time lag between pen manipulation on the tablet and movement of pen icon on HMD display.

The following three visuo-spatial tasks were administered in the traditional setting: a line bisection task, a figure copying task, and a figure cancellation task consisting of 20 triangles and 20 circles scattered on A4 paper (target "circle"). In the line bisection task, subjects were asked to mark the midpoint on each line. We analyzed the averaged percent deviation with respect to the true midpoint, defined as rightward and as a positive value in all trials. Following Levine's criteria (Levine, Warach, Benowitz \& Calvanio, 1986), we also graded the deviation: less than $9.9 \%$ as $0,10.0 \%$ to $39.9 \%$ as $1,40.0 \%$ to $69.9 \%$ as 2 , and more than $70.0 \%$ as 3 . In the figure copying task, subjects copied a daisy. We analyzed the area of parts omitted and scored it 0 if there were no omissions, 1 if less than the left-most $1 / 3$ was omitted, 2 if more than $1 / 3$ but less than $2 / 3$ was omitted, and 3 for more than $2 / 3$ omission. In the figure cancellation task, subjects were asked to cancel out all circles. We analyzed the number of targets canceled out and time taken. The same criteria were used for cancellation. We also calculated the performance score as a function of the number of canceled targets and time taken using the following formula: the number of canceled targets $\times$ the number of canceled targets / time taken. A higher score was considered to indicate a better performance.

\section{Tasks in HMD setting}

\section{1) Apparatus and experimental setting}

A head mount display (Eye-Trek FMD-250W, Olympus) (HMD) was used. HMD is a type of eye-ware, and only the task is displayed on it. Its screen resolution was
$653 \times 368$ pixels: the viewing area was $37.5^{\circ}$ (horizontal) $\times 21.7^{\circ}$ (vertical) of visual angle. Subjects operated a pen icon displayed on the HMD with a stylus on a tablet. We also used a tablet (intuos2 i-920 WACOM) with an A4size work space on which subjects conducted tasks with the stylus. These two apparatuses were connected to a lap top computer (Winbook WA2200C5, SOTEC) whose refresh rate and screen resolution was $60 \mathrm{~Hz}$ and $1024 \times 768$ pixels, respectively. Task stimulus for the HMD settings was made using a drawing application.

A schematic representation of the experimental settings is shown in Fig.1. The computerized stimulus was projected on the HMD through a down scan converter, allowing the experimenter to switch the projection on and off. The subjects wore the HMD and carried out the tasks with the stylus on the work area of the tablet. The top left corner and the bottom right corner of the work area of the tablet corresponded approximately to those of the HMD display.

\section{2) Task}

The HMD setting includes two sessions. Firstly, to familiarize themselves with the use of the HMD and the stylus, subjects carried out a series of trial line drawing and pointing tasks. In the line drawing task, subjects were instructed to draw a horizontal line while wearing the HMD. In the pointing task, they were asked to mark two black dots presented on the HMD display. Their familiarization was evident if they correctly drew a horizontal line and marked two dots. After familiarization, subjects 
Table 2. Directional attention behavioral questionnaire

\begin{tabular}{|c|c|c|c|}
\hline item & \multicolumn{3}{|c|}{ score } \\
\hline 1 to experience difficulty in finding people or objects on contralesional side & 0. none & 1. sometime & 2. frequently \\
\hline 2 to experience difficulty in looking towards contralesional side & 0. none & 1. sometime & 2. frequently \\
\hline 3 to forget to eat food on contralesional side of plate & 0. none & 1. sometime & 2. frequently \\
\hline 4 to forget to eat food on contralesional side of dish & 0. none & 1. sometime & 2. frequently \\
\hline 5 to forget to groom or shave on contralesional side of face & 0. none & 1. sometime & 2. frequently \\
\hline 6 to experience difficulty in adjusting contralesional side of sleeve & 0. none & 1. sometime & 2. frequently \\
\hline 7 to forget to use wheelchair brake on contralesional side & 0. none & 1. sometime & 2. frequently \\
\hline 8 to forget to use wheelchair foot-rest on contralesional side & 0. none & 1. sometime & 2. frequently \\
\hline 9 to start to transfer without taking foot off contralesional side of foot-rest & 0. none & 1. sometime & 2. frequently \\
\hline 10 to bump into something on contralesional side & 0. none & 1. sometime & 2. frequently \\
\hline 11 to experience difficulty in finding way towards contralesional side & 0. none & 1. sometime & 2. frequently \\
\hline 12 to be unaware of noise or people addressing him/her from contralesional side & 0. none & 1. sometime & 2. frequently \\
\hline \multirow[t]{2}{*}{13 to orient ipsilesional side when one is called } & 0. none & 1. sometime & 2. frequently \\
\hline & \multicolumn{3}{|c|}{$\operatorname{sum}(0 / 26)=$} \\
\hline
\end{tabular}

(adapted and revised from Ishiai, S. (1999) and Azouvi, P. et al. (2003))

carried out a figure cancellation task. The number of the targets (circles) and the distracters (triangles) was the same as that in the traditional setting. We used the cancellation task in the HMD setting because this cancellation task has several analytic components (Manly et al., 2009) and a higher sensitivity to attentional deficit (Azouvi et al., 2002). We applied the same analysis used in the traditional setting.

\section{Overall procedure}

Subjects were seated in front of the table. Firstly, they were asked to conduct tasks in the traditional setting: a line bisection task 5 times for each length, a figure cancellation task and a figure copying task. Then the tablet was placed and they carried out the familiarization task in the HMD setting. Finally, the figure cancellation task in the HMD setting was carried out.

\section{Behavior in $A D L$}

To assess the subjects' behavioral condition in ADL, we used two questionnaires. One was designed to assess the association of behavioral disorders with directional attentional deficit (Ishiai, 1999; Azouvi et al., 2003), for example "to bump into something on the contralesional side," and "to orient oneself to the ipsilesional side when one is called." 13 items were included and each item was scaled from 0 (never) to 2 (frequently) (Table 2). Therefore, the total score ranged from 0 to 26 . Another questionnaire was associated with general attentional deficit (Ponsford \& Kinsella, 1991), including "to be slow in movement," and "to miss important details in what he / she is doing." This questionnaire has 14 items scored from 0 (not at all) to 4 (always) for each item, and 0 to 56 as a total score (Table 3). Therapists who were in charge of the patients answered these questionnaires.

\section{Data analysis}

In the first analysis, we classified subjects into positive groups and negative groups on the basis of the cutoff score for each task: subjects who scored higher than 1 were positive and a 0 score was negative. Subjects showing attentional deficit on the tasks were identified as positive and those who didn't as negative. For the performance score in the figure cancellation task in both test settings, the cut-off score was the median of the performance score. We compared the scores of each behavioral questionnaire of the positive group with those of the negative group. Mann-Whitney's U test was applied for the comparison.

The second analysis was conducted to identify how much influence the HMD setting had on attention behavioral disorders. The traditional setting to HMD setting ratio was calculated for their performance score and compared with the total score of each attention behavioral questionnaire. Spearman's rank correlation coefficient was performed.

To learn whether HMD tasks could be an index of attention behavioral disorders, a third analysis examined variations in the results of tasks and attention behavioral problems at an individual level.

\section{Results}

\section{Positive group vs. negative group}

In all tasks performed, the directional attention behavioral score of the subjects who were diagnosed as positive was significantly higher: total score of the three visuo-spatial tests $(p=0.0009)$, line bisection ( $p=0.0435)$, copying $(p=0.0341)$, traditional cancellation (number of omits) $(p=0.0016)$, traditional cancellation (performance score) $(p=0.0051)$, and HMD cancellation (performance score) 
Table 3. General attention behavioral questionnaire

\begin{tabular}{|c|c|c|c|c|c|}
\hline item & \multicolumn{5}{|c|}{ score } \\
\hline 1 seemed lethargic (i.e. lacking energy) & 0 . Not at all & 1. Occasionally & 2. Sometimes & 3. Almost always & 4. Always \\
\hline 2 tired easily & 0 . Not at all & 1. Occasionally & 2. Sometimes & 3. Almost always & 4. Always \\
\hline 3 been slow in movement & 0 . Not at all & 1. Occasionally & 2. Sometimes & 3. Almost always & 4. Always \\
\hline 4 been slow to respond verbally & 0 . Not at all & 1. Occasionally & 2. Sometimes & 3. Almost always & 4. Always \\
\hline 5 performed slowly on mental tasks & 0 . Not at all & 1. Occasionally & 2. Sometimes & 3. Almost always & 4. Always \\
\hline 6 needed prompting to get on with things & 0 . Not at all & 1. Occasionally & 2. Sometimes & 3. Almost always & 4. Always \\
\hline 7 stared into space for long periods & 0 . Not at all & 1. Occasionally & 2. Sometimes & 3. Almost always & 4. Always \\
\hline 8 had difficulty concentrating & 0 . Not at all & 1. Occasionally & 2. Sometimes & 3. Almost always & 4. Always \\
\hline 9 been easily distracted & 0 . Not at all & 1. Occasionally & 2. Sometimes & 3. Almost always & 4. Always \\
\hline 10 been unable to pay attention to more than one thing at once & 0 . Not at all & 1. Occasionally & 2. Sometimes & 3. Almost always & 4. Always \\
\hline 11 made mistakes because he/she wasn't paying attention properly & 0 . Not at all & 1. Occasionally & 2. Sometimes & 3. Almost always & 4. Always \\
\hline 12 missed important details in what he/she is doing & 0 . Not at all & 1. Occasionally & 2. Sometimes & 3. Almost always & 4. Always \\
\hline 13 been restless & 0 . Not at all & 1. Occasionally & 2. Sometimes & 3. Almost always & 4. Always \\
\hline \multirow[t]{2}{*}{14 been unable to stick at an activity for very long } & 0 . Not at all & 1. Occasionally & 2. Sometimes & 3. Almost always & 4. Always \\
\hline & \multicolumn{5}{|c|}{$\operatorname{sum}(0 / 56)=$} \\
\hline
\end{tabular}

(adapted from Ponsford \& Kinsella. (1991))

(a) Directional attention behavior

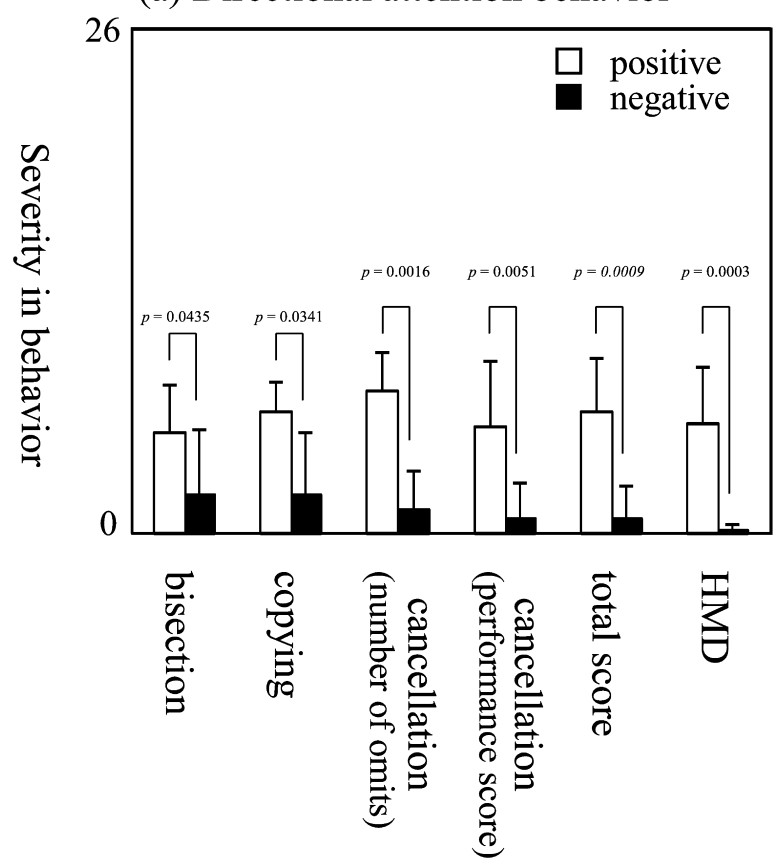

(b) General attention behavior

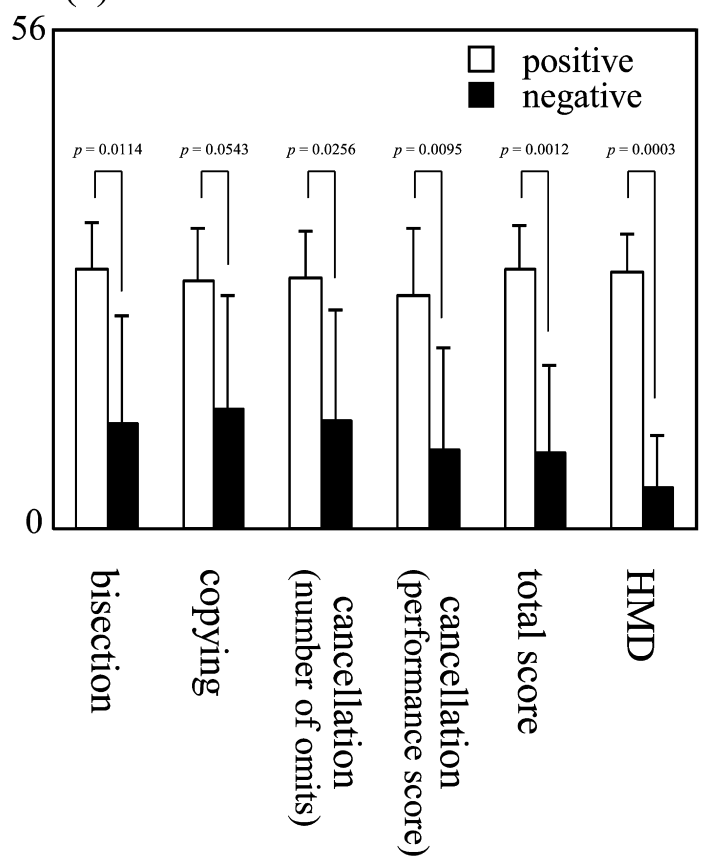

Fig. 2. Behavioral score of positive group and negative group for each task in (a) and (b). For both directional and general attention behavior, the positive group exhibits a much higher score than the negative group.

( $p=0.0003$ ) (see Fig. 2a). The same was true for the general attention behavioral score, except in the figure copying task, in which no difference between the two groups was found. In the other 5 tasks and indexes, the positive group achieved a significantly higher score than the negative group: total score of the three visuo-spatial tests ( $p=$ $0.0012)$, line bisection $(p=0.0114)$, traditional cancellation (number of omits) $(p=0.0256)$, traditional cancellation (performance score) $(p=0.0095)$, and HMD cancellation (performance score) $(p=0.0003)$ (see Fig. 2b).

\section{Traditional / HMD ratio and behavior}

Although all subjects showed lower performance scores in HMD tasks, the mean of the traditional / HMD ratio was 5.6, ranging from 2.0 to 15.5 . As shown in Fig. 3a, subjects with a significantly higher ratio achieved a higher score in the directional attention questionnaires $(r=0.78,<0.0005)$. A similar tendency was found in general attention behavior $(r=0.68,<0.005$; Fig. 3b). A separate correlation of the HMD task vs. each attentional behavior score and of traditional cancellation (perfor- 
(a) Directional attention behavior

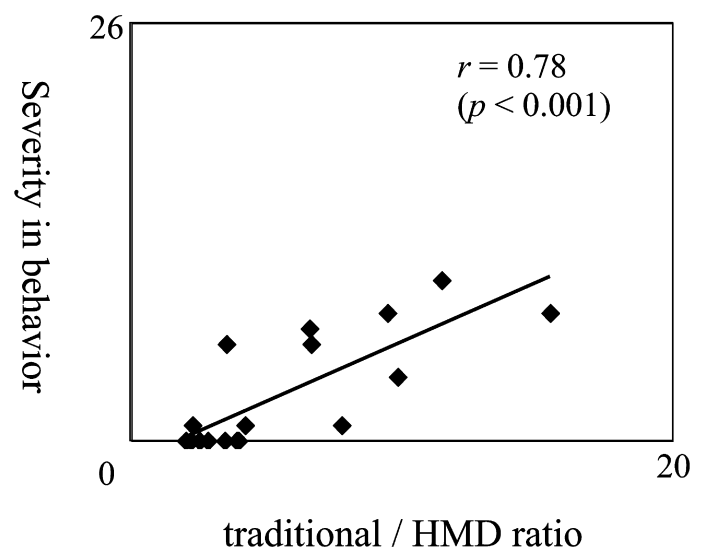

(b) General attention behavior

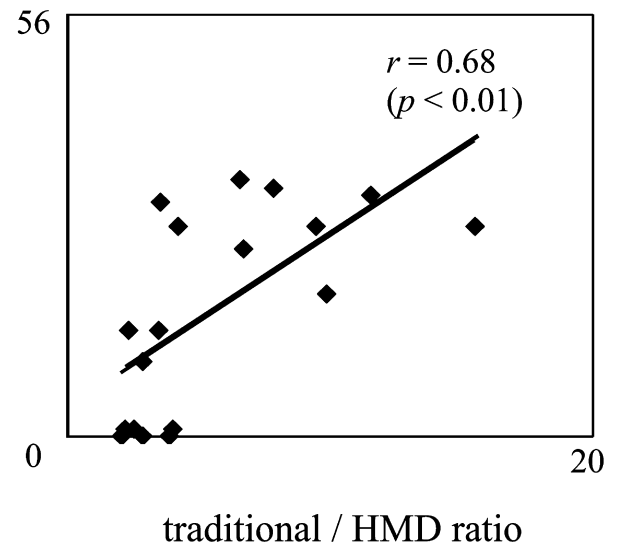

Fig. 3. Correlation between severity in behavior and traditional / HMD ratio in their performance score in the cancellation task in (a) and (b). In both behavioral scores, subjects who present higher ratio showed more severe behavioral problems. Each diamond represents one subject.

Table 4. Correlation between tasks and attentional behavior

\begin{tabular}{lll}
\hline & \multicolumn{2}{c}{ Attentional Behavior Score } \\
\cline { 2 - 3 } & Directional Attention & General Attention \\
\hline HMD & $\mathrm{r}=0.75(\mathrm{p}<0.01)$ & $\mathrm{r}=0.78(\mathrm{p}<0.01)$ \\
Cancellation (performance score) & $\mathrm{r}=0.65(\mathrm{p}<0.05)$ & $\mathrm{r}=0.56(\mathrm{p}<0.05)$ \\
Cancellation (number of omit) & $\mathrm{r}=0.84(\mathrm{p}<0.01)$ & $\mathrm{r}=0.58(\mathrm{p}<0.1)$ \\
Bisection & $\mathrm{r}=0.47(\mathrm{p}<0.1)$ & $\mathrm{r}=0.52(\mathrm{p}<0.1)$ \\
Copying & $\mathrm{r}=0.54(\mathrm{p}<0.05)$ & $\mathrm{r}=0.5(\mathrm{p}<0.1)$ \\
\hline$(* * p<0.01, * p<0.05$. n.s. : not significant $)$ &
\end{tabular}

mance score) vs. attentional behavior score are summarized in Table 4. The correlation between other traditional tasks, including cancellation as a function of the number of omits, bisection task and copying task and attentional behavioral questionnaires are also shown in Table 4. No significant correlation was found except in cancellation (number of omits) vs. directional attentional behavior $(r$ $=0.84,<0.01)$ and in the copying task vs. directional attentional behavior $(r=0.54,<0.05)$.

\section{Variations at the individual level}

We used the total score of the three visual-spatial tests and the performance score in the traditional cancellation task and in the HMD task as indexes for behavior deficit. For directional attention behavior, three subjects diagnosed as negative in the total score showed more than one behavioral problem. In the other three subjects, more than one directional attention behavior problem was evident, whereas they were diagnosed as negative through the performance score in the traditional cancellation task. On the other hand, only one subject who was diagnosed as negative in the HMD task showed directional attention behavior, but his score in the behavioral questionnaire was extremely low (1/26) (Fig. 4a).
8 subjects diagnosed as negative in the total score of the desk-tasks displayed general attention behavior problems. In 7 subjects, general attention behavioral problem were evident despite their performance score on the traditional cancellation task being below the cut-off score. For the HMD task, three subjects who scored higher in the general attention questionnaires were diagnosed as positive. The remaining 6 subjects diagnosed as negative in the HMD task showed general attention behavioral problems, however their score was not higher than the other subjects (Fig. 4b).

\section{Discussion}

\section{Theoretical framework of the HMD setting}

As described in the introduction, we assumed that environmental differences, static in desk-task settings and dynamic in ADL settings, could lead to the discrepancy whereby patients show abnormalities in ADL but not in desk-task settings. To evaluate this assumption, we newly devised a more dynamic task environment than the traditional task environment using a HMD and a tablet, based on the theory of frame of reference proposed by Karnath (1994). 
(a) Directional attention behavior

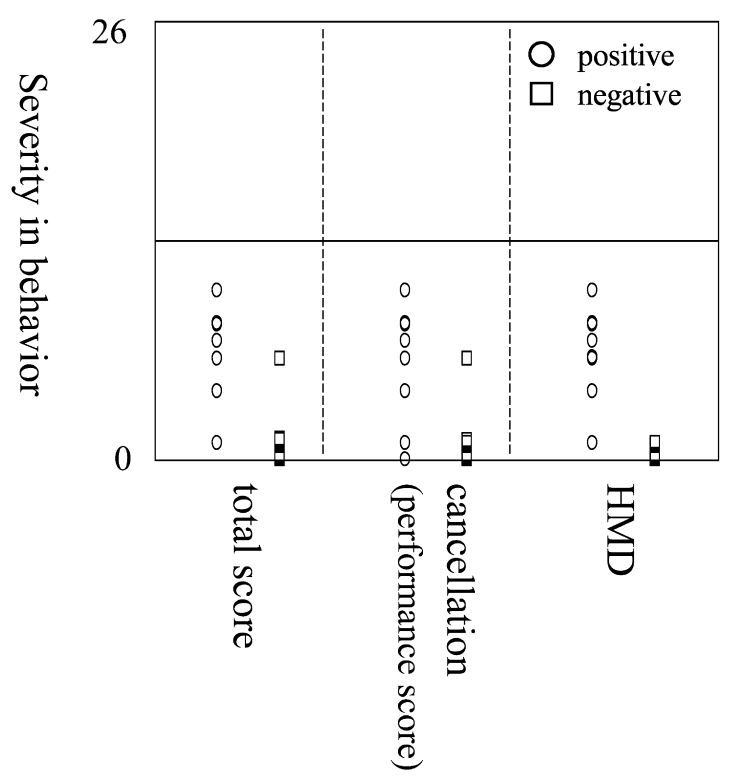

(b) General attention behavior
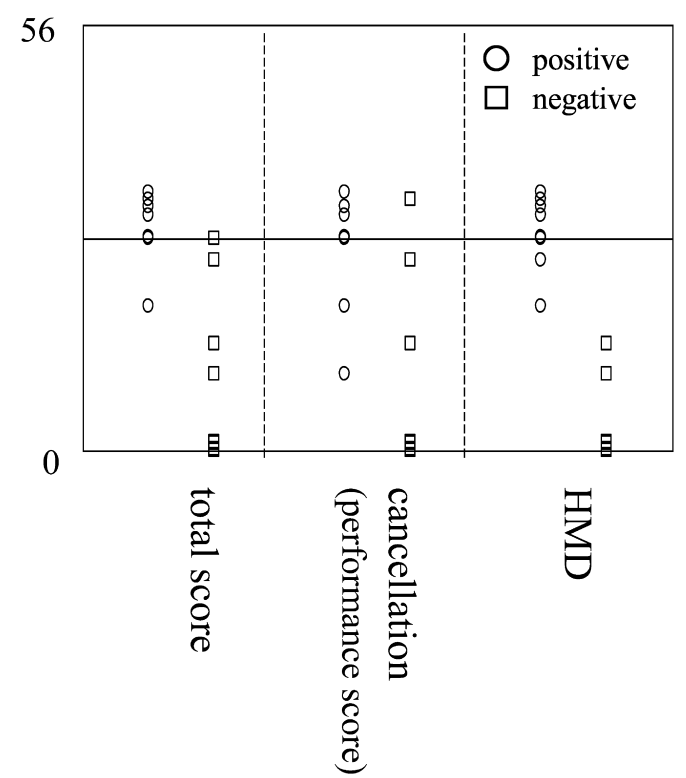

Fig. 4. Severity in behavior at the individual level. Left row of each column represents subjects( $(\circ)$ diagnosed as positive, marks in the right row $(\square)$ corresponds to negative. (a) For directional attention behavior, all but one subject diagnosed as negative by HMD task did not show behavioral problems. (b) For general attention behavior, subjects diagnosed as negative by the HMD task exhibited lower behavioral score.

According to Karnath (1994), Karnath, Fetter, and Niemeier (1998), and Karnath and Dieterich (2006), humans behave accurately in space by integrating incoming sensory information such as vision, proprioception, and vestibular sensation, and these should be integrated into an egocentric reference frame. This successive and concurrent process is the key concept of the HMD setting. We postulated that a more dynamic task environment could be accomplished by taxing such a process during testing.

Subjects wear HMD and handle a stylus in the HMD setting. As shown in Fig.1, the only things subjects can directly see on the HMD display are task stimulus and the pen icon, which represents the real pen they manipulate on the tablet. Subjects cannot see the movement of their hand or upper extremity while operating the pen. Indirect vision of the hand trajectory induces conflicting visual and proprioceptive signals (Clower \& Boussaoud, 2000). This experimental environment must strongly encourage subjects to integrate proprioceptive sensation from their arm and finger movements with vision on the HMD display while testing. In other words, subjects need to reorganize their work space by integrating the proprioceptive space and visual space while performing the HMD task (Vakalopoulos, 2007).
Relation between the results of the HMD task and attention behavior

The most interesting result of this study was that in both directional and general attentional behavior, almost all the subjects who were classified into the negative group in the HMD task did not display attentional disturbance in ADL or vice versa. This implies that the HMD setting has a similar cognitive feature to that of the ADL environment. As mentioned earlier, the ADL environment is so dynamic that sensory information relevant to concurrent activity must be integrated (Deouell, Sacher \& Soroker, 2005; Thompson \& Sanderson, 2008). According to this idea, the present results support our postulation that the HMD setting induces a dynamic situation and requires subjects to perform much more sensory integration more than in the traditional setting. In the HMD setting, subjects have to rely more on proprioception to adapt to sensory-motor conflict. In fact, Thompson and Sanderson (2008) have pointed out that one of the reasons why results of the traditional desk-task were not fully congruent with conditions in ADL was that the desk-task was less dynamic. Tilikete, Rode, Rossetti, Pichon, Li, and Boisson (2001) reported that reorganization of the spatial framework led to amelioration in ADL performance. Several studies concur with those reports (Karnath, Fetter \& Niemeier, 1998; Karnath \& Dieterich, 2006; Rorden, Karnath \& Driver, 2001; Niemeier \& Karnath, 2003). As in the HMD environment, in ADL, humans have to adapt 
to a constantly changing dynamic experience by correctly selecting internal and external cues relevant to the subsequent action. This environmental similarity between HMD and ADL may have led to the apparent correlation between performance in the HMD setting and behavioral disorders in ADL.

Another possible interpretation is provided by Burgess (2000). He insisted that daily activity consists of multi-tasking situations. In the HMD setting, subjects were encouraged to conduct the cancellation task as well as adapt to the HMD environment. However, the concept of multi-tasking doesn't fully explain the present results because the same results should have been obtained from the traditional task setting, as this setting involves multitasking aspects to some degree.

In the present study, only the cancellation task was used in the HMD setting. Considering that there are several neuropsychological tests available, further studies incorporating different desk-tasks should be carried out.

\section{Applicability in clinical settings}

The present results reveal that although all subjects marked lower performance scores in HMD tasks compared to traditional cancellation tasks, subjects who scored above the cut-off in the HMD tasks seldom exhibited both directional and general attention behavioral problems. This implies that HMD tasks are a more sensitive tool than traditional neuropsychological tests for the evaluation of presence / absence of attentional deficit in ADL if evaluated by means of the cut-off score used in the present study.

In recent years, the number of studies using an HMD or a virtual environment is increasing. Knight and Baber (2007) reported the influence of HMD on posture. Cho, $\mathrm{Ku}$, Jang, Kim, et al. (2002) and Cho, Ku, Jang, Lee, et al. (2002) developed an attention training system using an HMD and an HMD device for people with impaired vision (Culham, Chabra \& Rubin, 2004). In addition, HMD could be used to train patients with visuo-spatial problems (Castiello, Lusher, Burton, Glover \& Disler, 2004; Kim et al., 2004; Baheux, Yoshizawa, Tanaka, Seki \& Handa, 2005; Baheux, Yoshizawa, Seki \& Handa, 2006; Glover \& Castiello, 2006). In clinical practice in occupational therapy, Weiss, Naveh, \& Katz (2003) and Katz et al. (2005) introduced a training system for patients with spatial neglect to navigate road traffic safely. Almost all of these studies were addressing the issues of therapy or intervention as well as simple evaluation (the presence / absence of a certain symptom). To the best of our knowledge, few studies have addressed the issue of clinical applicability through establishment of an index for attentional behavioral problems in everyday life.

As seen in Fig. 4, subjects who were classified as negative according to their total score and performance score in traditional cancellation showed attentional behavioral problems. By these criteria, the total score of the three visuo-spatial tasks and performance score in traditional cancellation can't be an index of behavioral problems: no data exists to the contrary. On the other hand, HMD differentiated those subjects. As mentioned by Sasaki et al. (2005), changing the environment under which tasks are conducted rather than changing the task stimulus itself could account for the present results.

In conclusion, the present preliminary study would appear to demonstrate that tasks carried out in the HMD setting can function as a useful index of attentional behavioral problems.

\section{References}

Azouvi, P., Samuel, C., Louis-Dreyfus, A., Bernati, T., Bartolomeo, P., Beis, JM., et al. (2002). Sensitivity of clinical and behavioral tests of spatial neglect after right hemisphere stroke. Journal of Neurology, Neurosurgery \& Psychiatry. 73, 160-166. [Medline] [CrossRef]

Azouvi, P., Olivier, S., de Montety, G., Samuel, C., LouisDreyfus, A., \& Tesio, L. (2003). Behavioral Assessment of Unilateral Neglect: Study of the Psychometric Properties of the Catherine Bergego Scale. Archives of Physical Medicine and Rehabilitation. 84, 51-57. [Medline] [CrossRef]

Baheux, K., Yoshizawa, M., Tanaka, A., Seki, K., \& Handa, Y. (2005). Diagnosis and rehabilitation of hemispatial neglect patients with virtual reality. Technology and Health Care. 13, 245-260. [Medline]

Baheux, K., Yoshizawa, M., Seki, K., \& Handa, Y. (2006). Virtual reality pencil and paper tests for neglect: a protocol. Cyberpsychology Behavior. 9, 192-195. [Medline] [CrossRef]

Burgess, PW. (2000). Strategy application disorder: the role of the frontal lobes in human multitasking. Psychological Research. 63, 279-288. [Medline] [CrossRef]

Castiello, U., Lusher, D., Burton, C., Glover, S., \& Disler, P. (2004). Improving left hemispatial neglect using virtual reality. Neurology. 62, 1958-1962. [Medline]

Cho, BH., Ku, J., Jang, D., Kim, S., Lee, Y., Oh, M., et al. (2002). The effect of virtual reality cognitive training for attention enhancement. Cyberpsychology \& Behavior. 5, 129-137. [Medline]

Cho, BH., Ku, J., Jang, D., Lee, J., Oh, M., Kim, H., et al. (2002). Clinical test for attention enhancement system. Stud Health Technol Inform. 85, 89-95. [Medline]

Clower, DM., \& Boussaoud, D. (2000). Selective use of perceptual recalibration versus visuomotor skill acquisition. Journal of Neurophysiology. 84, 2703-2708. [Medline]

Culham, LE., Chabra, A., \& Rubin, GS. (2004). Clinical performance of electronic, head-mounted, low-vision devices. Ophthalmic and Physiological Optics. 24, 281-290. [Medline] [CrossRef]

Deouell, LY., Sacher, Y., \& Soroker, N. (2005). Assessment of 
spatial attention after brain damage with a dynamic reaction time test. Journal of the International Neuropsychological Society. 11, 697-707. [Medline] [CrossRef]

Glover, S., \& Castiello, U. (2006). Recovering space in unilateral neglect: A neurological dissociation revealed by virtual reality. Journal of Cognitive Neuroscience. 18, 833-843. [Medline] [CrossRef]

Ishiai, S., editor.(1999). Behavioral inattention test. Japanese edition (C1999. Tokyo, Japan: Shinkoh Igaku Shuppan.

Karnath, HO. (1994). Disturbed coordinate transformation in the neural representation of space as the crucial mechanism leading to neglect. Neuropsychological Rehabilitation. 4, 147-150. [CrossRef]

Karnath, HO., Fetter, M., \& Niemeier, M. (1998). Disentangling gravitational, environmental, and egocentric reference frames in spatial neglect. Journal of Cognitive Neuroscience. 10, 680-690. [Medline] [CrossRef]

Karnath, HO., \& Dieterich, M. (2006). Spatial neglect - a vestibular disorder? Brain. 129, 293-305. [Medline] [CrossRef]

Katz, N., Ring, H., Naveh, Y., Kizony, R., Feintuch, U., \& Weiss, PL. (2005). Interactive virtual environment training for safe street crossing of right hemisphere stroke patients with spatial neglect. Disability and Rehabilitation. 27, 1235-1244. [Medline] [CrossRef]

Kim, K., Kim, J., Ku, J., Kim, DY., Chang, WH., Shin, DI., et al. (2004). A virtual reality assessment and training system for unilateral neglect. CyberPsychology Behavior. 7, 742-749. [Medline] [CrossRef]

Knight, JF., \& Baber, C. (2007). Effect of head-mount displays on posture. The Journal of the Human Factors and Ergonomics Society. 49, 797-807. [CrossRef]

Levine, DN., Warach, JD., Benowitz, L., \& Calvanio, R. (1986). Left spatial neglect: effect of lesion size and premorbid brain atrophy on severity and recovery following right cerebral infraction. Neurology. 36, 362-366. [Medline]

Manly, T., Dove, A., Blows, S., George, M., Noonan, MP., Teasdale, TW., et al. (2009). Assessment of spatial neglect: scoring star cancellation performance from video recordings - method, reliability, benefit, and normative data. $\mathrm{Neu}$ ropsychology. 23, 519-528. [Medline] [CrossRef]

Niemeier, M., \& Karnath, HO. (2003). Stimulus-driven and voluntary saccades are coded in different coordinate systems. Current Biology. 13, 585-589. [Medline] [CrossRef]

Ponsford, J., \& Kinsella, G. (1991). The use of a rating scale of attentional behaviour. Neuropsychological Rehabilitation. 1, 241-257. [CrossRef]

Rorden, C., Karnath, HO., \& Driver, J. (2001). Do neck-proprioceptive and caloric-vestibular stimulation influence covert visual attention in 49 normals, as they influence visual neglect? Neuropsychologia. 39, 364-375. [Medline] [CrossRef]

Sasaki, T., Sengoku, Y., Nakajima, S., Sugama, K., \& Kitajima, H. (2005). The correlation between the results of the line bisection test with the test seat placed in different positions regarding the midsagittal plane of the USN patients and their ADL. The journal of Japanese association of occupational therapists, 24, 584-592.

Thompson, MB., \& Sanderson, PM. (2008). Multisensory integration with a head-mount display: sound delivery and self-motion. The Journal of the Human Factors and Ergonomics Society. 50, 789-800. [CrossRef]

Tilikete, C., Rode, G., Rossetti, Y., Pichon, J., Li, L., \& Boisson, D. (2001). Prism adaptation to rightward optical deviation improves postural imbalance in left-hemiparetic patients. Current Biology. 11, 524-528. [Medline] [CrossRef]

Vakalopoulos, C. (2007). Unilateral neglect: a theory of proprioceptive space of a stimulus as determined by the cerebellar component of motor efference copy (and is autism a special case of neglect). Medical Hypotheses. 68, 574 600. [Medline] [CrossRef]

Weiss, PL., Naveh, Y., \& Katz, N. (2003). Design and testing of a virtual environment to train stroke patients with unilateral spatial neglect to cross a street safely. Occupational Therapy International. 10, 39-55. [Medline] [CrossRef] 